\title{
THE
}

\section{Optical Measurements of Capillary-Gravity Wave Spectra Using a Scanning Laser Slope Gauge}

\author{
Erik J. Bock \\ Tetsu Hara \\ University of Rhode Island, thara@uri.edu
}

Follow this and additional works at: https://digitalcommons.uri.edu/gsofacpubs

Terms of Use

All rights reserved under copyright.

\section{Citation/Publisher Attribution}

Bock, E.J., \& Hara, T. (1995). Optical Measurements of Capillary-Gravity Wave Spectra Using a Scanning Laser Slope Gauge. J. Atmos. Oceanic Technol. 12, 395-403. doi: 10.1175/1520-0426(1995)0122.0.C0;2. Available at: https://doi.org/10.1175/1520-0426(1995)0122.0.C0;2

This Article is brought to you for free and open access by the Graduate School of Oceanography at DigitalCommons@URI. It has been accepted for inclusion in Graduate School of Oceanography Faculty Publications by an authorized administrator of DigitalCommons@URI. For more information, please contact digitalcommons-group@uri.edu. 


\title{
Optical Measurements of Capillary-Gravity Wave Spectra Using a Scanning Laser Slope Gauge
}

\author{
ERIK J. BOCK \\ Woods Hole Oceanographic Institution, Woods Hole, Massachusetts \\ TETSU HARA \\ Graduate School of Oceanography, University of Rhode Island, Narragansett, Rhode Island
}

(Manuscript received 6 August 1993, in final form 14 June 1994)

\begin{abstract}
A description of a new scanning laser slope gauge (SLSG) is given and the results obtained from both laboratory wind-wave tank and field measurements are presented. The device relies on the measurements of two components of surface slope to compute spatial and temporal lags that are used to estimate the full threedimensional slope spectrum. The device is capable of resolving frequencies up to $34.7 \mathrm{~Hz}$ and wavelengths in the range between $7.9 \times 10^{-3}$ and $3.08 \times 10^{-1} \mathrm{~m}$. The technique makes use of a two-dimensional laser scanner that samples the perimeter of a circle of $0.154-\mathrm{m}$ diameter (an unfilled aperture). Both laboratory and field results indicate the device is well suited to measure the full three-dimensional spectra of capillary-gravity waves and is capable of providing ground-truthing measurements for the verification of remotely sensed ocean surface features.
\end{abstract}

\section{Introduction}

Characterization of ocean surface waves is important to understand many aspects of air-sea interaction. Heat, momentum, and mass fluxes are all influenced by surface waves. In particular, the knowledge of gas fluxes are fundamental to questions regarding the partitioning of carbon dioxide and other greenhouse gases between the atmosphere and the ocean. Additionally, the remote sensing of ocean surface from scatterometer, real aperture radar, or synthetic aperture radar relies on scattering of electromagnetic waves by short capillary-gravity waves. This remote sensing is important for the global observation of wind speed (for use in weather and ocean circulation models) and various wave-wave and wave-current interactions.

Previous attempts to measure the spatial spectrum use various techniques, including stereo-photogrammetry (Banner 1989), laser scanning (Hwang 1989, 1992; Barter et al. 1990; Lee et al. 1992), and digital image processing (Jähne and Riemer 1990). Only the techniques described in the last three references are capable of making two- or three-dimensional spectral estimates in the capillary-gravity range of wavelengths. The digital image-processing technique is convenient and accurate in the laboratory, but its use in situ has

Corresponding author address: Dr. Erik J. Bock, Department of Applied Ocean Physics and Engineering, Woods Hole Oceanographic Institution, Woods Hole, MA 02543. not been reported. The two-dimensional scanning laser slope gauge used by Barter et al. (1990) is both costly to use and physically quite large; this latter aspect introduces significant flow distortions in the wind field, and hence the wave field, at the point of measurement.

Recently the authors have developed a low-profile scanning laser slope gauge (SLSG) (Martinsen and Bock 1992; Bock and Hara 1992). The instrument has been designed to be integrated with a research catamaran to acquire unobstructed measurements of capillary-gravity wave spectra as the catamaran is towed through features of interest, with less flow distortion compared with past devices. Preliminary laboratory tests with mechanically generated waves have revealed that the instrument is capable of measuring three-dimensional wavenumber-frequency spectra of capillarygravity waves (Bock and Hara 1992). The instrument has since been significantly improved to reduce the noise level. The dynamic range of the new SLSG allows for gathering spectral information of waves with wavelengths between $7.9 \times 10^{-3}$ and $3.08 \times 10^{-1} \mathrm{~m}$ and frequencies up to $34.7 \mathrm{~Hz}$. The instrument has been deployed successfully both in a laboratory wind-wave tank and in situ.

In section 2 we describe the optomechanics, electronics, data-acquisition hardware, and calibration of the SLSG. Sections 3 and 4 describe the calculation of the slope spectrum and its error analysis. Results obtained from both laboratory and field experiments are discussed in section 5 . 


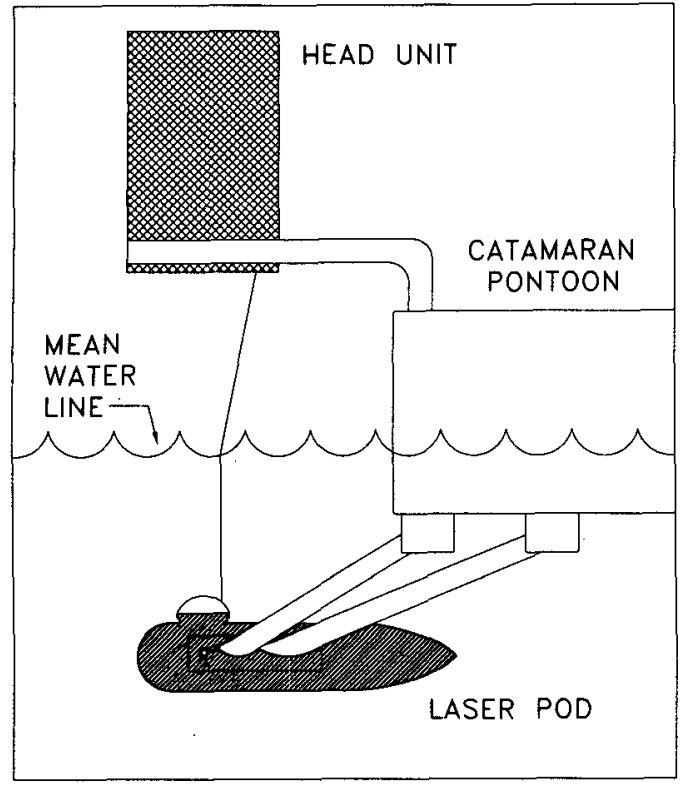

FIG. 1. Schematic configuration of the laser pod and head unit mounted to the pontoon of the catamaran.

\section{Sensor design and data-collection strategy}

The SLSG consists of two subassemblies, namely, a laser pod that produces a collimated, positionable light source and a head unit that is an optical receiver. Both units were designed to be rigidly attached to a catamaran with the laser pod affixed beneath the waterline and the head unit above the waterline. The basic configuration is depicted in Fig. 1. In this arrangement, the optical axis of the system is determined by the relative positions of the laser pod and head unit.

As the catamaran is towed through the water, the SLSG unobstructively measures the local slope of the free surface relative to the instrument. The laser beam is scanned to extract spatiotemporal data for the measurement of full three-dimensional spectra. As illustrated in Fig. 2, the beam is refracted by the slope of air-sea interface owing to the difference in refractive indices. The amount of angular refraction is uniquely related to the local slope $(\tan \alpha)$ of the free surface by Snell's law,

$$
n_{1} \sin \alpha=n_{2} \sin \beta,
$$

and geometry gives

$$
\gamma=\beta-\alpha,
$$

where $n_{1}$ is equal to 1.33 for seawater, and $n_{2}$ is equal to 1.00 for air. The amount of deflection of the beam is then measured by the head unit. The beam is first focused by an aspheric lens onto a diffusive element, which then scatters the beam toward an array of four photodiodes. The two components ( $x$ and $y$ components) of the deflection are obtained in real time from

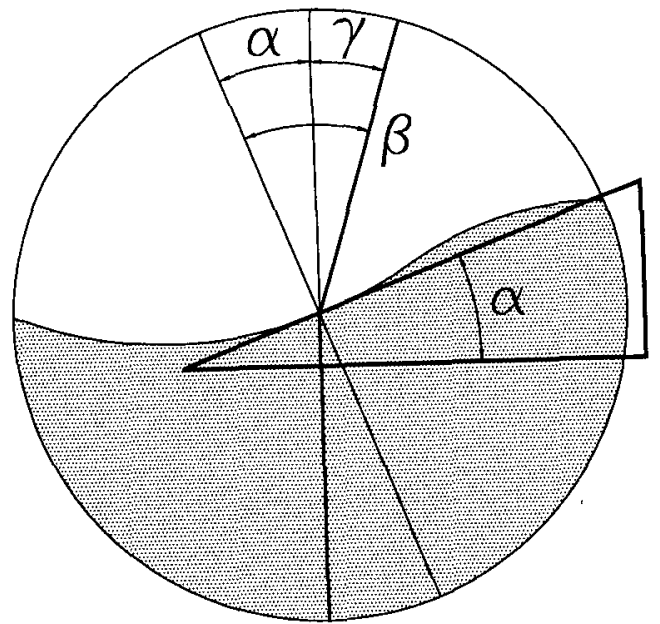

FIG. 2. Beam geometry at the air-sea interface. The vertical line represents the optical axis. The incoming beam is parallel to the optical axis and refracts at the interface because of the local slope, $\tan \alpha$. The beam is deflected away from the optical axis by an amount $\gamma$.

an array of photodiodes in the head unit by a centroiding algorithm. Finally, a look-up table is used to relate measured beam deflection to actual surface slope ( relative to the instrument).

\section{a. Laser pod}

The laser pod, shown in Fig. 3, contains a 10-mW $\mathrm{CW}$ helium-neon ( $\mathrm{He}-\mathrm{Ne}$ ) laser, HN1; a reflecting prism, $\mathrm{P} 1$; reflecting mirrors, $\mathrm{M} 1$ and $\mathrm{M} 3$; a rotating mirror, M2; and a custom aspheric lens, L1. All the components, except for the lens, are secured to a common tray, which is attached to one of the end caps of an underwater pressure housing. The tray facilitates optical alignment outside of the tube in addition to keeping the alignment intact whenever the tray is removed from the tube for diagnostic purposes. 'The housing is faired with fiberglass shapes $F 1$ and F2 that reduce drag as it is towed through the water.

Beam propagation through the laser pod is described by the following sequence. The He-Ne laser emits a 0.8mm-diameter TEM $_{00}$ (transverse electric-magnetic) beam measured at the $1 / e^{2}$ irradiance points. The light emerging from the laser is reflected upward and then

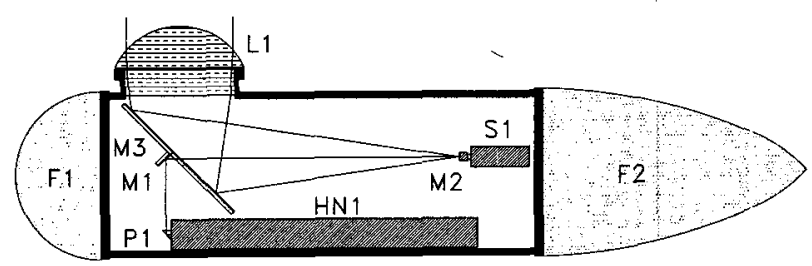

FIG. 3. Laser pod, with helium-neon laser HN1; prism P1; mirrors $\mathrm{M} 1, \mathrm{M} 2$, and $\mathrm{M} 3$; motor $\mathrm{S} 1$; lens $\mathrm{L} 1$; and fairings $\mathrm{F} 1$ and $\mathrm{F}$.2. 


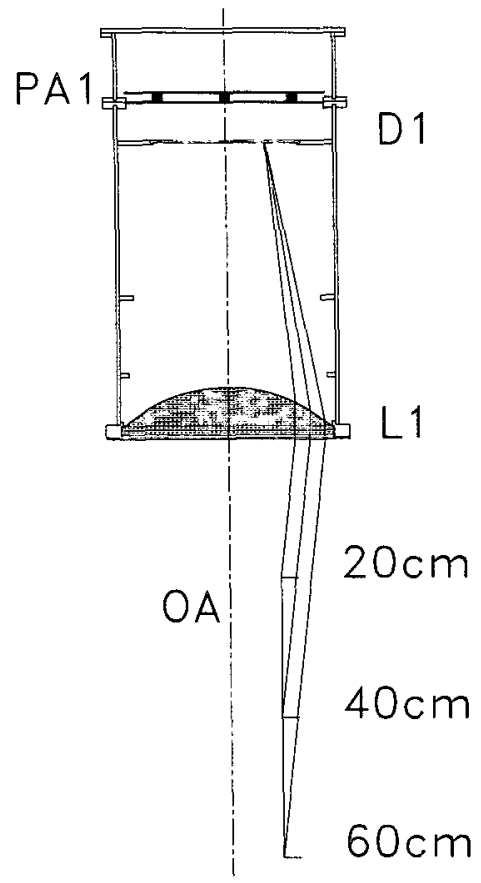

FIG. 4. Head unit, with lens L1; diffusing plate DI; and photodiode array PAl. The optical axis (OA) is at the center of the head unit. Representative rays are drawn to indicate the range over which the water-to-head unit distance $d$ typically varies. The circular scan pattern is axisymmetric about the optical axis.

back by the combination of $\mathrm{P} 1$ and M1. The beam strikes rotating mirror $\mathrm{M} 2$, which is attached to a motor at a slightly oblique angle. The motor, spinning at 25000 $\mathrm{rpm}$, traces out a hollow cone of light, performing a two-dimensional scan. The reflected scanning beam propagates forward, down the long axis of the laser pod back to M3, which redirects the beam $90^{\circ}$ up to the aspheric lens L 1 . The lens, with air on one side and seawater on the other, collimates the scan pattern so that all rays are parallel with the optical axis as they emerge from the lens. After the beam is refracted by the air-sea interface, it enters the head unit, and its angular deflection is measured by the spot centroiding algorithm described below.

\section{b. Head unit}

A schematic of the head unit is shown in Fig. 4. Along the upward direction of the optical path, components in the head unit include a plano-convex lens, L1; a diffusive plate at the focal plane of the lens, D1; and a printed circuit board containing four silicon photodiodes and analog signal-conditioning electronics, PA1. The four photodiodes are mounted in a square pattern centered around the optical axis, with a diagonal distance between diodes of $0.18 \mathrm{~m}$. The photodiodes are used in pairs by pairing the diagonal couples. Photodiodes $\mathrm{A}$ and $\mathrm{C}$ constitute the bow-stern pair and $B$ and $D$ constitute the starboard-port pair.
The diffusive plate D1, positioned at the focal plane of the lens L1, acts as a quasi-Lambertian surface operating in transmission. Its purpose is to forward-scatter the laser radiation into a broad Gaussian profile. Detection of scattered light at a distance of $0.2 \mathrm{~m}$ above $\mathrm{D} 1$ is used to determine the position of the beam. The algorithm yields the $x$ and $y$ coordinates of the spot position according to

$$
x=\frac{I_{\mathrm{A}}-I_{\mathrm{C}}}{I_{\mathrm{A}}+I_{\mathrm{C}}-2 I_{\mathrm{L}}}
$$

and

$$
y=\frac{I_{\mathrm{B}}-I_{\mathrm{D}}}{I_{\mathrm{B}}+I_{\mathrm{D}}-2 I_{\mathrm{L}}}
$$

where $I_{\mathrm{A}}, I_{\mathrm{B}}, I_{\mathrm{C}}$, and $I_{\mathrm{D}}$ are the intensities measured at each of the photodiodes and $I_{\mathrm{L}}$ is the intensity of the ambient light. Ambient light $I_{\mathrm{L}}$ is obtained by an analog sample-and-hold circuit that detects and retains the intensities at the photodiode pairs during the instant when the laser beam is intentionally blocked at a location around the perimeter of the circle. With this scheme, the degradation of signal caused by strong ambient light can be minimized as long as the ambient light does not change significantly during the period of scan (i.e., $2.4 \times 10^{-3} \mathrm{~s}$ ). This condition is met under almost all circumstances, and it is clear from the raw data when it is violated, so that contaminated data may be discarded.

\section{c. Sensor control, data acquisition, and modes of operation}

A functional block diagram showing the electrical interfaces between the sensor, the control electronics, and the data-acquisition system is illustrated in Fig. 5. The acquisition system includes a generic $80486 \mathrm{DX} 2$ 66 personal computer with a 12-bit CIO-AD16JR-AT

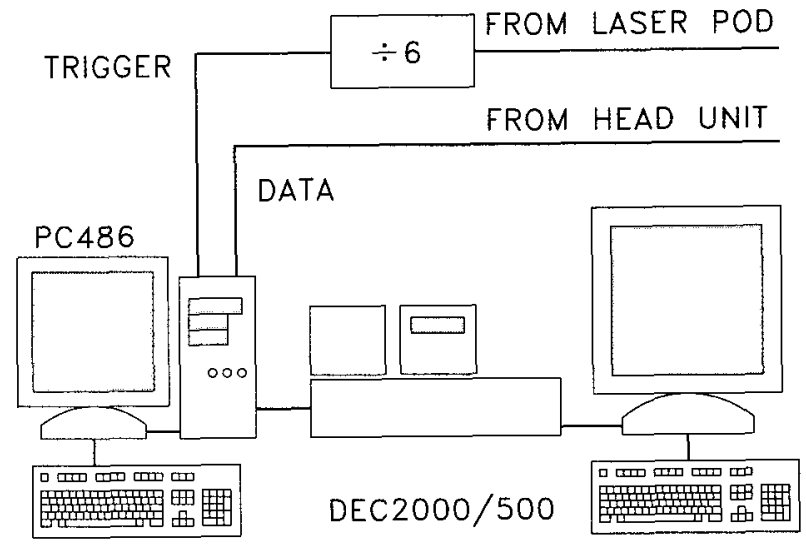

FIG. 5. Block diagram of the data-acquisition system. Timing is determined by the laser pod, and data collected with the PC486 is stored via ethernet on the DEC2000/500 workstation. 
data-acquisition card (Computer Boards, Inc.), a DEC Station $2000 / 500$, and some digital logic to perform timing functions.

The laser pod emits a digital signal each time the beam is blocked for ambient light cancellation; this happens every $2.4 \times 10^{-3} \mathrm{~s}$. A digital divide-by- $N$ circuit is used to reduce the frequency of scanning. In theory, the value of $N$ can be set between 2 and 255 , but to match the spatial and temporal scales of measurement, a value of 6 was chosen in most cases. This gives a sampling frequency of $69.4 \mathrm{~Hz}$ (period equal to $1.44 \times 10^{-2} \mathrm{~s}$ ). Every $1.44 \times 10^{-2} \mathrm{~s}$, the data-acquisition system is triggered to poll 237 readings of surface slope around the perimeter of scan. This corresponds to a spatial separation of $2.04 \times 10^{-3} \mathrm{~m}$ between points. Hours of data are collected and then transmitted over an ethernet connection and stored on a fixed disk drive of the DEC 5000. This task is repeated until the end of data collection.

\section{Data analysis}

\section{a. Calibration}

Before use, the response of the head unit was calibrated to determine the relationship between the output signal and the surface slope. It was decided that we first perform numerical ray tracing to generate a look-up table relating the output signal and the surface slope for all possible ray combinations. In this process we have assumed that the diffuser scatters the beam with a constant coefficient of angular scatter $D$ (ratio between incident and refracted angles) and spreads the ray into a Gaussian distribution with standard deviation $\sigma_{d}$. The refraction coefficient $D$ and the standard deviation $\sigma_{d}$ are determined empirically so as to minimize the difference between the ray-tracing results and actual surface slope calibrations, which have been performed at three different distances $d$ (as typified in Fig. 4 ) between the start of angular refraction and the head unit.

Figure 6a shows the output from the head unit for a distance of $d=0.6 \mathrm{~m}$, which roughly corresponds to the largest distance between the water surface and the head unit in the field operation. The beam is deflected in a conical sweep at five different angles corresponding to surface slopes of $0,0.087,0.176,0.268$, and 0.364 . Clearly, the ray-tracing model reproduces the actual output very accurately. The deviation of the measurements from the ray-tracing calculation follows a Gaussian distribution, as shown in Fig. 6b.

\section{b. Computation of three-dimensional spectra}

We start with the definition of the wave slope spectrum. Spectral density functions are usually estimated through finite Fourier transforms of sampled data. However, in order to estimate the three-dimensional spectrum in this manner, it is necessary to sample the
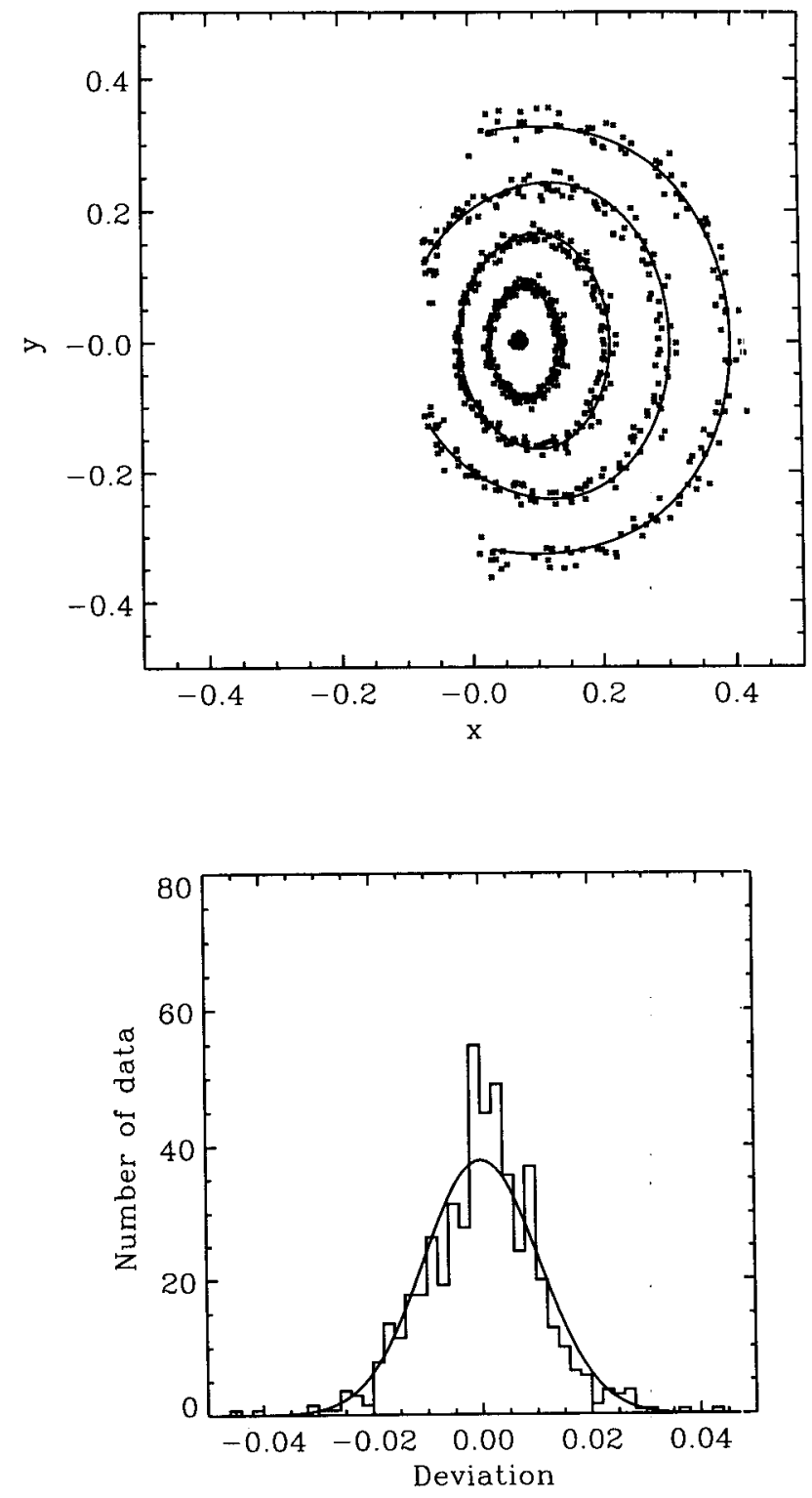

FIG. 6. (a) Output from the head unit for conical sweeps of beam, corresponding to surface slope of $0,0.087,0.176,0.268,0.364$. Solid lines indicate estimates by ray-tracing calculation. Distance between the start of beam refraction and the head unit is $0.6 \mathrm{~m}$. (b) Histogram of the deviation of output from the ray-tracing calculatior: and Gaussian fit (solid line).

data in a dense three-dimensional domain (two spatial dimensions and one temporal dimension). This demands a very high speed laser scan and a high sampling rate. Instead, we estimate the spectrum through autocorrelation functions. This method requires only that the spatial and temporal lags fill the three-dimensional domain. We achieve this goal by scanning the laser beam along the perimeter of a circle in space and sampling the wave slope around the perimeter. As shown in Fig. 7, proper combinations of the sampled data 
along the circle can fill the two-dimensional lag space. The spatial resolution in the lag domain is comparable to that in the physical domain, that is, the spatial sampling rate. By repeating this measurement at a given frequency, we can also obtain any spatiotemporal lags, with the temporal resolution in the lag domain corresponding to the sampling rate.

Let us define $x, y$ to be horizontal coordinates and $z$ to be the vertical, upward coordinate. We denote wave height by $\zeta$ and the two components of the wave slope by

$$
\frac{\partial \zeta}{\partial x}=s_{1}(x, y, t) \quad \frac{\partial \zeta}{\partial y}=s_{2}(x, y, t) .
$$

The autocorrelation functions of the surface slope are then defined to be

$$
\begin{array}{r}
R_{i}(\xi, \eta, \tau)=\left\langle s_{i}(x, y, t) s_{i}(x+\xi, y+\eta, t+\tau)\right\rangle, \\
i=1,2,
\end{array}
$$

where angle brackets denote ensemble average. The two-sided, three-dimensional, wavenumber-frequency slope spectrum is calculated as

$$
\begin{array}{r}
S_{i}\left(k_{1}, k_{2}, \omega\right)=\frac{1}{8 \pi^{3}} \iiint R_{i} e^{-i k_{1} \xi-i k_{2} \eta-i \omega \tau} d \xi d \eta d \tau, \\
i=1,2
\end{array}
$$

for the $i$ th component of slope, where $\omega$ is frequency and $k_{1}, k_{2}$ are the $x, y$ components of the wavenumber. They can be written as

$$
k_{1}=k \cos \theta, \quad k_{2}=k \sin \theta,
$$

where $k$ is the absolute wavenumber and $\theta$ the wave direction. The total slope spectrum is the sum of the two components,

$$
S\left(k_{1}, k_{2}, \omega\right)=S_{1}\left(k_{1}, k_{2}, \omega\right)+S_{2}\left(k_{1}, k_{2}, \omega\right),
$$

with the relation that

$$
S_{1}=S \cos ^{2} \theta, \quad S_{2}=S \sin ^{2} \theta .
$$

The spectrum may be written in cylindrical coordinates as

$$
S\left(k_{1}, k_{2}, \omega\right) d k_{1} d k_{2} d \omega=S(k, \theta, \omega) k d k d \theta d \omega .
$$

The latter form is more convenient in data analyses. The two-sided wavenumber spectrum $(0<\theta<2 \pi)$ is obtained by integrating the three-dimensional spectrum in frequency domain:

$$
S(k, \theta)=\int_{-\infty}^{\infty} S(k, \theta, \omega) d \omega .
$$

In summary, the entire analysis consists of the following steps.

1) Sampling: Surface slope is measured at 237 discrete points along a circle in $2.4 \times 10^{-3} \mathrm{~s}$. This sampling is repeated at a fixed rate up to $69.4 \mathrm{~Hz}$.
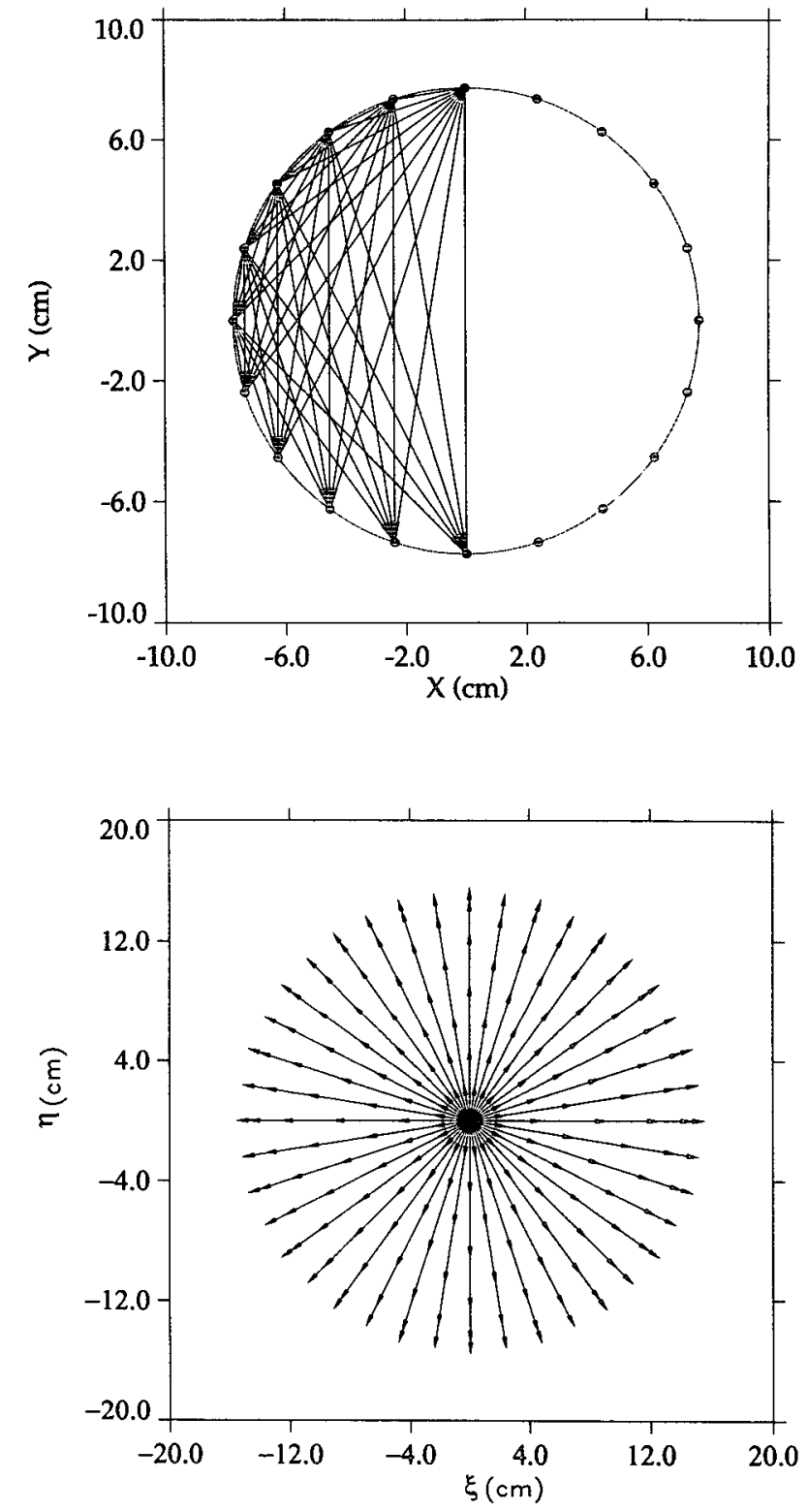

FIG. 7. By combining sampling points along the perimeter of a circle in (a), it is possible to fill the spatial lag domain in (b).

2) Calculation of spatiotemporal lags: Spatial lags are obtained by combining appropriate points along the perimeter of the circle, as shown in Fig. 7. In the lag calculation we assume that each scan is completed instantaneously, that is, the surface is assumed to be frozen during each scan. From our scanning slope gauge measurement, we have $0.5(237 \times 236)=27966$ discrete spatial lags with length between $2.04 \times 10^{-3}$ and $1.54 \times 10^{-1} \mathrm{~m}$, including 237 discrete angles. In the time domain, lags equal to any multiple of the frame rate are possible. In the calculations below, 64 discrete time lags were used. The ensemble average in (6) is replaced by the time average. The length of the 
averaging varies depending on the type of measurement.

3) Windowing: Chosen windows are applied both in time and in space in order to reduce the side lobes. In the following examples we have used cosine-square tapering in two-dimensional wavenumber domain (which corresponds to Hanning window in rectangular grids). In the time domain regular Hanning window has been chosen.

4) Three-dimensional discrete Fourier transform: Theoretically we can resolve frequencies up to the Nyquist, which is one-half of the frame rate, and wavenumbers in the range between 20 and $1500 \mathrm{rad} \mathrm{m}^{-1}$. Current design of the window, however, only allows the wavenumber resolution up to $800 \mathrm{rad} \mathrm{m}^{-1}$.

\section{Error analysis}

There are many possible sources of error in our technique. However, significant errors can be summarized in the following six categories.

\section{a. Bias due to pitch and roll of the instrument}

As mentioned before, the measurement of the surface slope is in the frame of reference moving with the instrument. The effect of the pitch and roll of the instrument appears in two ways. First, the real surface slope value is not exactly the sum of the measured surface slope and the slope of the instrument itself. Second, the projection of the scanned beam on the water surface is slightly elongated from the true circle, hence, the wavelength of the observed waves can be underestimated. The second effect also appears due to the mean slope of the real ocean surface produced by long gravity waves. All these effects are at most of the order of $S_{i} S_{s}^{2}$ or $S_{s}^{2} S_{s}$, where $S_{i}$ is the slope of the instrument and $S_{s}$ is the slope of the surface. Since $S_{i}$ never exceeds about 0.1 (by visual observation), these errors can be always safely neglected for our purpose.

\section{b. Bias due to calibration}

We have completed a look-up table relating the output and the surface slope at all possible heights of the water surface relative to the SLSG. In a laboratory experiment, the water level stays within a narrow range and is known. However, in a field measurement, the inclusion of the changing water level is complicated because independent measurement of the water level must be incorporated. The catamaran was therefore equipped with three capacitance wave gauges to achieve this goal. In practice, however, we have found that using a look-up table at a mean height $(d=40 \mathrm{~cm})$ does not degrade the measurement significantly as long as the spectrum is averaged over a time period much longer than the dominant gravity wave period. (This is because the plano-convex lens in the head unit was designed so that the dependence of the output on $d$ is minimized.) The results were found to differ by no more than $1 \mathrm{~dB}$ based on spectral calculations using our field data. Of course, if one is interested in the variation of capillary-gravity wave spectra over a time period comparable to that of dominant gravity waves, a full calibration including the independent measurement of water surface level and platform attitude is necessary.

\section{c. Effect of finite scan speed}

With the current design of the laser pod, a potentially serious source of error comes from the fact that the beam does not sweep a circle instantly but over a finite duration of $2.4 \times 10^{-3} \mathrm{~s}$, which smears high-frequency waves since the spectral calculation assumes a frozen surface during one scan. This error becomes more serious when the slope gauge is towed at a high speed when the apparent frequency of short waves increases significantly. To analyze these effects, we have used numerically synthesized periodic waves. In Fig. 8 we show calculated one-dimensional (omnidirectional) wavenumber spectra for simulated waves at $k=600$

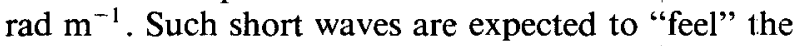
effect of finite scan speed rather strongly. Since the simulated waves are of a single wavenumber, if the sampling scheme truly "freezes" the ocean surface (i.e., solid line), the calculated spectrum is peaked at a single wavenumber and exhibits numerical sidelobes consistent with the applied window. Note that the sidelobe decays much faster than typical wavenumber spectra $\left(\propto k^{-1.5}\right)$. When the actual finite scan speed is included in the calculation (dash-dot line), the spectral shape is almost identical to the case of instantaneous scanning. Even with a constant tow speed of $1 \mathrm{~m} \mathrm{~s}^{-1}$

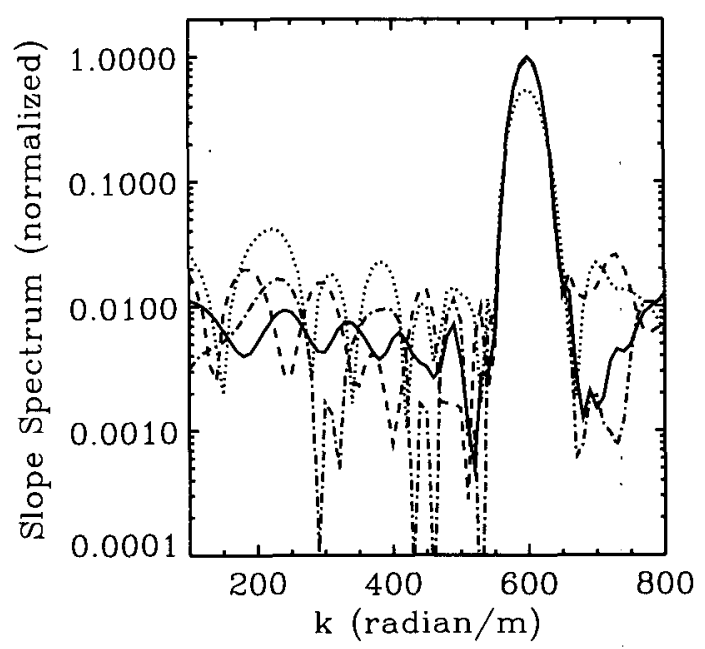

FIG. 8. One-dimensional wavenumber slope spectrum for numerically simulated waves at $k=600 \mathrm{rad} \mathrm{m}^{-1}$. Solid line: instantaneous scan, zero tow speed; dash-dot line: actual scan, zero tow speed; dashed line: actual scan, $1 \mathrm{~m} \mathrm{~s}^{-1}$ tow speed; dotted line: actual scan, $4 \mathrm{~m} \mathrm{~s}^{-1}$ tow speed. 


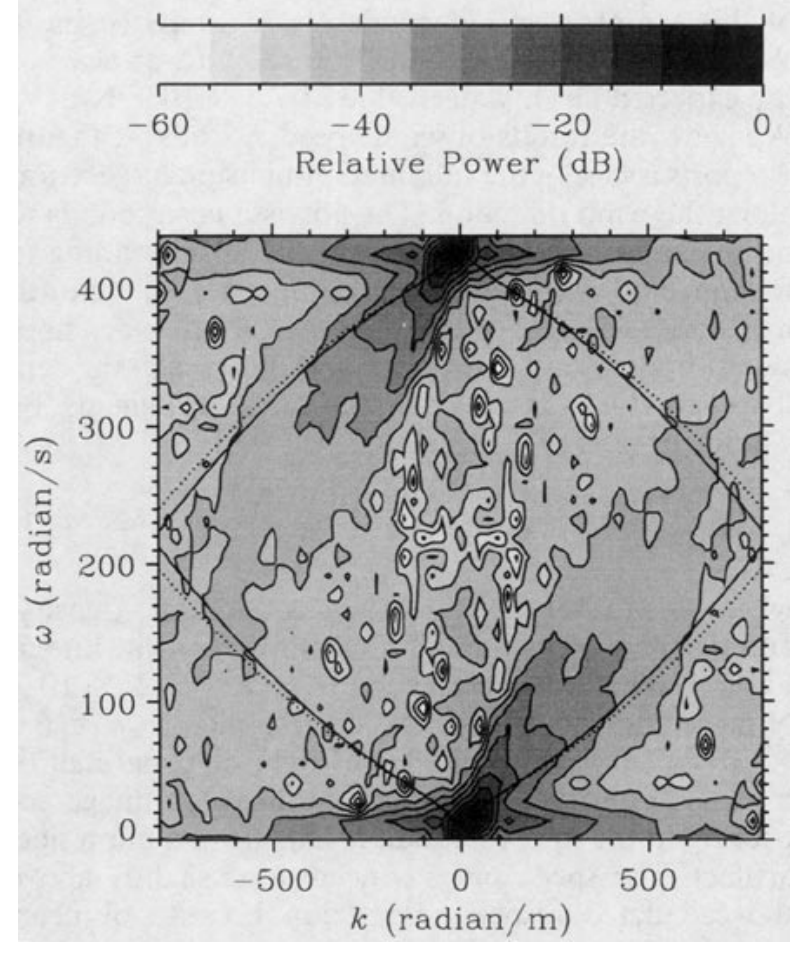

Fig. 9. Two-dimensional slope spectrum $(k-\omega)$ for waves propagating on clean water in along-wind direction. Solid lines and dashed lines denote the capillary-gravity dispersion relation for surface tension $7.3 \times 10^{-2}$ and $6.0 \times 10^{-2} \mathrm{~N} \mathrm{~m}^{-1}$, respectively. Laboratory measurements in a wind-wave tank. Wind speed $7.5 \mathrm{~m} \mathrm{~s}^{-1}$. Relative power $0 \mathrm{~dB}$ corresponds to $10^{-3} \mathrm{~m}^{2}$.

(dashed line), the error is reasonably small. However, the case of a large tow speed ( $4 \mathrm{~m} \mathrm{~s}^{-1}$, dotted line) shows that the spectral estimate is reduced by as much as $40 \%$ near the peak. Therefore, the measurement of real ocean surface slope spectra obtained at large tow speeds can be contaminated. These results indicate that the scanning slope gauge must be deployed at a moderate tow speed ( up to $1 \mathrm{~m} \mathrm{~s}^{-1}$ ) to measure the ocean wave spectra accurately. Accordingly, all the field measurements have been performed at less than $1 \mathrm{~m} \mathrm{~s}^{-1}$.

\section{d. Statistical random error}

A confidence level estimate has been performed using the actual data obtained from field measurements. The raw, three-dimensional spectral estimate has been found to have a Gaussian distribution, with the standard deviation inversely proportional to the square root of the time averaging period for each estimate. The deviation can also be reduced by averaging the result over a range of wave direction $\theta$ and/or temporal frequency $\omega$. We have found that the $95 \%$ confidence level is roughly $\pm 1 \mathrm{~dB}$ if the following averages are taken:

1) three-dimensional spectrum in $(k, \theta, \omega)$ averaged over $1 \mathrm{~h}$, with angular resolution of $30^{\circ}$;
2) two-dimensional wavenumber spectrum in $(k$, $\theta$ ) averaged over $10 \mathrm{~min}$, integrated over all frequencies, with angular resolution of $10^{\circ}$;

3) one-dimensional wavenumber spectrum in $k$ averaged over $1 \mathrm{~min}$, integrated over all frequencies and all directions.

\section{e. Data dropout}

Data dropout occurs by two reasons; one being a locally large surface slope that refracts the beam outside the head unit, the other being large gravity waves that yield water surface levels either below the top of the laser pod or above the bottom of the head unit. In the laboratory, data dropout was rarely observed even at high wind speeds. On the other hand, in the field experiments, data dropout was mainly due to the second reason, that is, gravity waves, and the dropout rate increased with wind speed. At wind speed $5 \mathrm{~m} \mathrm{~s}^{-1}$, the dropout was usually below $3 \%$, while the rate was as large as $50 \%$ at wind speed $9 \mathrm{~m} \mathrm{~s}^{-1}$. Although spectral calculations have been performed on data obtained from high wind cases after removing all the data dropout period, high data dropout rate can potentially bias the spectral estimate. This is because data dropout tends to occur at a particular phase of long waves, depending

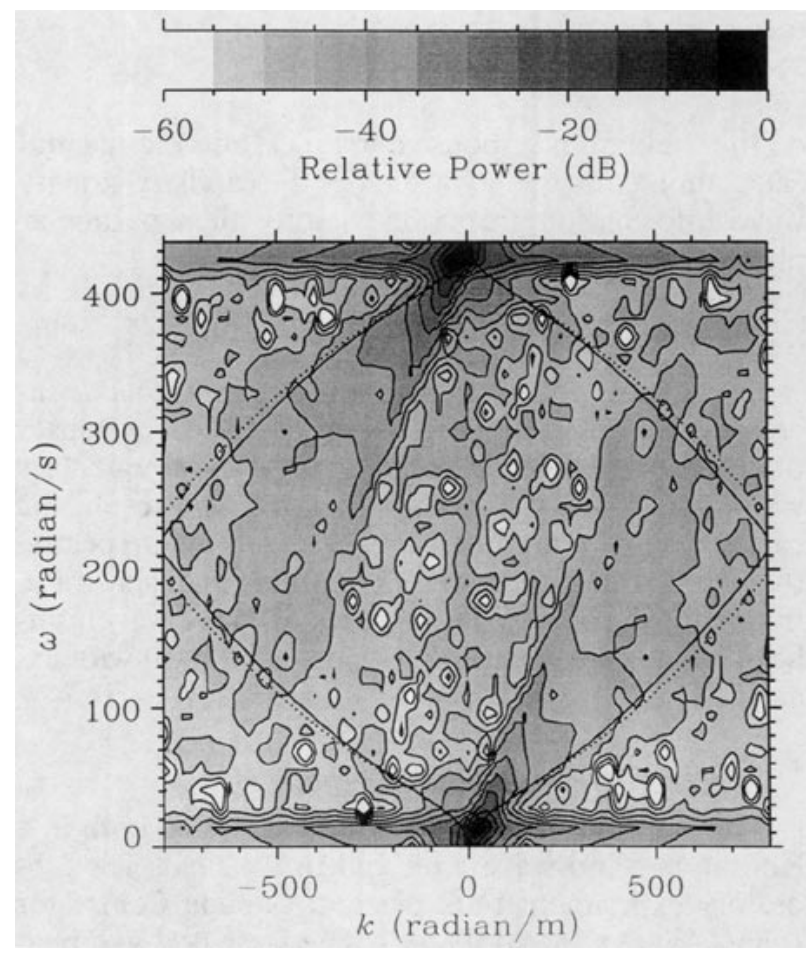

Fic. 10. Two-dimensional slope spectrum $(k-\omega)$ for waves propagating on a $1-\mu \mathrm{M}$ solution of Triton $\mathrm{X}-100^{\circ}$ in along-wind direction. Solid lines and dashed lines denote the capillary-gravity dispersion relation for surface tension $7.3 \times 10^{-2}$ and $6.0 \times 10^{-2} \mathrm{~N} \mathrm{~m}^{-1}$, respectively. Laboratory measurements in a wind-wave tank. Wind speed $7.5 \mathrm{~m} \mathrm{~s}^{-1}$. Relative power $0 \mathrm{~dB}$ corresponds to $10^{-3} \mathrm{~m}^{2}$. 


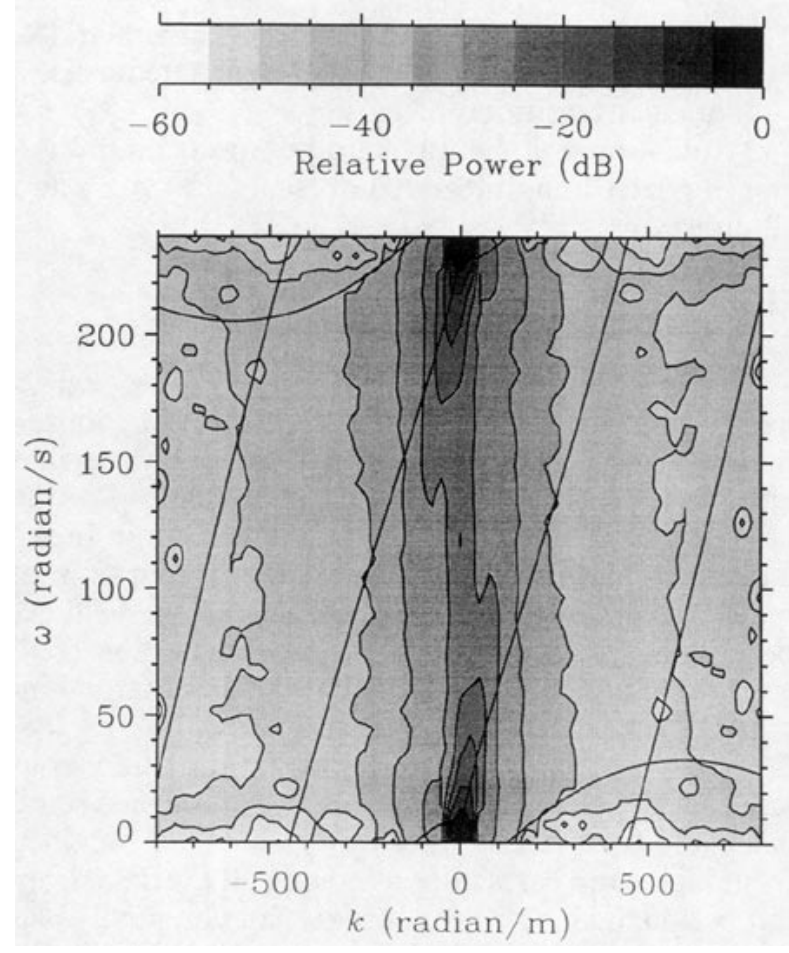

FIG. 11. Two-dimensional slope spectrum $(k-\omega)$ for waves propagating in along-wind direction. Solid lines denote the capillarygravity dispersion relation for clean water including the Doppler shift by tow speed of $0.3 \mathrm{~m} \mathrm{~s}^{-1}$. Field measurements. Wind speed $6 \mathrm{~m} \mathrm{~s}^{-1}$. Relative power $0 \mathrm{~dB}$ corresponds to $10^{-3} \mathrm{~m}^{2}$.

on the catamaran response dynamics, and the spectral calculation consequently excludes the capillary-gravity wave information from the vicinity of a particular phase of the long wave.

\section{f. Numerical sidelobes}

It has been found that as the wavenumber $k$ increases, the three-dimensional spectrum decays faster than the numerical sidelobes at very low frequencies where long gravity waves are present. As a result, the calculation of two-dimensional wavenumber spectra, that is, the integration of three-dimensional spectra in frequency domain, may be biased at lower wavenumbers. Details are discussed in the next section with examples.

\section{Laboratory and field experiments}

The scanning laser slope gauge was tested both in a laboratory wind-wave tank and in the field. The laboratory experiment took place at Canada Centre for Inland Waters in April 1993. The laser pod was held below the wave tank, and the beam was aimed up through the glass bottom of the tank to mimic the situation depicted in Fig. 1.

In the first set of experiments, distilled water was used as a substrate for the waves. The surface tension

of this sample was independently measured using a Wilhelmy plate and torsion balance and was close to the expected clean water value of $7.3 \times 10^{-2} \mathrm{~N} \mathrm{~m}^{-1}$. We show the results of wind speed $7.5 \mathrm{~m} \mathrm{~s}^{-1}$. Figure 9 reports a slice of the full three-dimensional spectrum along the wind direction. The abscissa corresponds to positive and negative wavenumber (corresponding, to forward and backward propagating waves), the ordinate corresponds to angular frequency. The black lines superimposed on the diagram are solutions to the capillary-gravity dispersion relation formulated by Thomson (1871):

$$
\omega^{2}=\frac{\sigma k^{3}}{\rho}+g k
$$

where $\sigma$ is the surface tension, $\rho$ is the liquid density, and $g$ is the gravitational constant. The solid line at lower wavenumber corresponds to $\sigma=7.3 \times 10^{-2}$ $\mathrm{N} \mathrm{m}^{-1}$, and the dotted line corresponds to $\sigma=6.0$ $\times 10^{-2} \mathrm{~N} \mathrm{~m}^{-1}$. Only the lower right diagonal half of this plot need be considered, the mirrored image appearing in the upper left half results from a numerical artifact. The spectrum is concentrated slightly above the calculated dispersion relation because of nearsurface drift current. The spreading of the spectrum in the vertical direction (in frequency) is due to the Doppler shift introduced by longer gravity waves. The high spectral level at large wavenumbers and very low frequencies is due to the sidelobes in $k$ domain associated with long gravity waves. Although the sidelobes decay roughly $-40 \mathrm{~dB}$ from the peak, the real spectrum decays to even lower levels. The calculation of the twodimensional spectrum (integrated over all frequencies) can be performed avoiding this sidelobe range. This is possible since the real spectrum near the dispersion relation is clearly separated from the sidelobe, except at very low wavenumbers.

The second set of experiments used a solution of Triton $X-100^{\circledR}$ (Rohm and Haas Inc.), with concentration of about $1 \mu \mathrm{M}$ to measure waves propagating on a liquid in the presence of a finite surface dilational viscoelasticity. Figure 10 reports the two-dimensional spectrum of waves propagating in the wind direction. The same power scale was used as in Fig. 10. Overall, the power is lower, being consistent with the increased rate of viscous dissipation owing to the inclusion of

TABLE 1. Main features of scanning laser slope gauge.

Scan diameter

Minimum wavelength

Maximum wavelength

Frame frequency

Nyquist frequency

Error estimate

Wind condition

Data dropout
$0.154 \mathrm{~m}$

$7.9 \times 10^{-3} \mathrm{~m}\left(800 \mathrm{rad} \mathrm{m}^{-1}\right)$

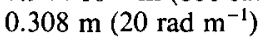
$69.4 \mathrm{~Hz}\left(436 \mathrm{rad} \mathrm{s}^{-1}\right)$ $34.7 \mathrm{~Hz}\left(218 \mathrm{rad} \mathrm{s}^{-1}\right)$ $<1 \mathrm{~dB}$ $<9 \mathrm{~m} \mathrm{~s}^{-1}$

$<3 \%$ at wind speed $5 \mathrm{~m} \mathrm{~s}^{-1}$ $<50 \%$ at wind speed $9 \mathrm{~m} \mathrm{~s}^{-1}$ 
dilational surface viscoelasticity. Because of the Doppler smearing by long waves, the difference of the dispersion relations between clean and slicked surfaces cannot be resolved.

Finally we show an example from field experiments off Cape Hatteras performed in June 1993. Note that the sampling rate has been reduced to $46.3 \mathrm{~Hz}$ in order to reduce the data rate. The same $k-\omega$ plot in the wind direction is shown in Fig. 11 at wind speed $6 \mathrm{~m} \mathrm{~s}^{-1}$. Since measurements were performed at a tow speed of roughly $0.3 \mathrm{~m} \mathrm{~s}^{-1}$, the theoretical dispersion relation has been Doppler shifted accordingly. At very low wavenumbers, the spectrum is still concentrated near the dispersion relation. On the other hand, for wavenumbers larger than $200 \mathrm{rad} \mathrm{m}^{-1}$, the Doppler shift due to long gravity waves is so strong that the wave energy spreads over all frequencies up to our sampling frequency of $46.3 \mathrm{~Hz}$. Again we observe the sidelobes propagating from long gravity waves at very low frequencies. When the spectrum is integrated over the frequency domain to avoid this sidelobe region, the estimate of the wavenumber spectrum is expected to be biased by no more than $1 \mathrm{~dB}$ for wavenumbers above $100 \mathrm{rad} \mathrm{m}^{-1}$.

\section{Concluding remarks}

A new scanning laser slope gauge has been developed that is capable of measuring the three-dimensional capillary-gravity wave spectra. Important features of the instrument are summarized in Table 1. The laboratory measurements show that the resolution of the instrument is high enough to quantify the reduction of the spectrum from surfactant effects. The field results of measuring capillary-gravity wavenumber spectra are of sufficient accuracy so as to provide ground-truthing measurements for microwave radar remote sensing at wind speeds up to about $9 \mathrm{~m} \mathrm{~s}^{-1}$. Our current efforts include further analysis of these experimental data to study the dynamics of wind-generated short waves under various environmental conditions.

Acknowledgments. This work was supported as part of the Office of Naval Research Accelerated Research Initiative entitled "High Resolution" under Grant N00014-91-J-1770. This is Woods Hole Oceanographic Institution contribution No. 8448.

\section{REFERENCES}

Banner, M. L., I. S. F. Jones, and J. C. Trinder, 1989: Wavenumber spectra of short gravity waves. J. Fluid Mech., 198, 32 I-344.

Barter, J., K. Beach, D. Kwoh, M. Ludwig, H. Rungaldier, J. Schatzman, I. Stonich, R. Wagner, and A. Williams, 1990: TRW surface truth measurement system. TRW Internal Rep. 53563-6005, $107 \mathrm{pp}$.

Bock, E. J., and T. Hara, 1992: Optical measurements of ripples using a scanning laser slope gauge, part II: Data analysis and interpretation from wave tank and field experiments. Proc., Optics of the Air-Sea Interface: Theory and Measurements, San Diego, CA, SPIE, $1749,272$.

Hwang, P. A., 1989: A scanning optical height-and-slope sensor for spatial measurements of wind-generated surface waves. STC Tech. Rep. 3099, 63 pp.

_ 1992: Optical measurements of short wave modulation by surface currents. Proc., Optics of the Air-Sea Interface: Theory and Measurements, San Diego, CA, SPIE, 1749, 216.

Jähne, B., and K. S. Riemer, 1990: Two-dimensional wave number spectra of small-scale water surface waves. J. Geophys. Res., 95, $11531-11546$.

Lee, P. H. Y., J. D. Barter, K. L. Beach, C. L. Hindman, B. M. Lake, H. Rungaldier, J. C. Shatzman, J. C. Shelton, R. N. Wagner, A. B. Williams, R. Yee, and H. C. Yuen, 1992: Recent advances in ocean surface characterization by a scanning laser slope gauge. Proc., Optics of the Air-Sea Interface: Theory and Measurements,

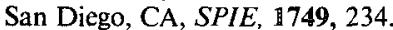

Martinsen, R. J., and E. J. Bock, 1992: Optical measurements of ripples using a scanning laser slope gauge, part I: Instrumentation and preliminary results. Proc., Optics of the Air-Sea Interface: Theory and Measurements, San Diego, CA, SPIE, 1749, 258.

Thomson, W., 1871: Hydrokinetic solutions and observations. Phil. Mag., 42, 362-374. 